\title{
Un codice di Federico Veterani alla Biblioteca Universitaria di Anversa
}

\author{
FABIO DELLA SCHIAVA
}

Antwerpen, Universiteitsbibliotheek, MAG-P 60.5 is the oldest manuscript held by the Special Collections department of the University of Antwerp. Because of its bibliographical value, specific attention has already been devoted to it in an article published in Scriptorium forty years ago. However, it failed to identify the scribe, the dedicatee and the occasion for which the manuscript had been created. Starting from a reconsideration of some neglected texts on the flyleaf of the manuscript and a fresh paleographical analysis, this article suggests to attribute it to the hand of Federico Veterani, a scribe for Federico da Montefeltro at the end of the fifteenth century.

Il codice Antwerpen, Universiteitsbibliotheek, MAG-P 60.5 è un manoscritto pergamenaceo di piccolo formato che raccoglie le satire di Giovenale e di Persio e l'Ars poetica di Orazio. Prima di approdare alla sua attuale collocazione, il codice era conservato nella biblioteca delle Universitaire Faculteiten Sint-Ignatius di Anversa (UFSIA), istituzione fondata nel 1852 dalla Compagnia di Gesù e confluita nel 2003, insieme al Rijksuniversitair Centrum Antwerpen (RUCA) e alla Universitaire Instelling Antwerpen (UIA), nella nuova Universiteit Antwerpen (UA). Nella sua antica collocazione, il codice portava la segnatura UFSIA P 1/1, con la quale Jan Gijsel ne segnalò l'alto valore bibliografico in un breve contributo apparso su Scriptorium: come mi conferma Tom Deneire, il manoscritto costituisce ancora oggi il pezzo più antico delle raccolte librarie dell'università. ${ }^{1}$ Gijsel offre una valutazione dei testi ivi contenuti per mezzo di un confronto con le rispettive edizioni critiche, soffermandosi in particolare sul testo di Giovenale. Inoltre, lo studioso fornisce una dettagliata descrizione esterna del codice, di cui passa in rassegna gli elementi paratestuali (decorazioni, segni di lettura,

\footnotetext{
* Ringrazio per le loro preziose indicazioni Irene Ceccherini, Fulvio Delle Donne, Giuseppe Marcellino, Marco Petoletti e Xavier Van Binnebeke.

1 J. Gijsel, "Un manuscrit inconnu des Satires de Juvénal et de Perse et de l'Art poétique d'Horace", Scriptorium 33.2 (1979), 266-270 (doi: 10.3406/scrip.1979.1143). Una copia digitale del manoscritto è disponibile all'indirizzo internet https://anet.uantwerpen.be/digital/opacuantwerpen/uapreciosa/dg:ua:791/N.
} 
interventi di correzione e note di possesso) allo scopo di ricostruirne la storia. $^{2}$

Sulla base della decorazione dispiegata nel frontespizio (TAVOLA 1), Gijsel ipotizza che il codice provenga dall'Italia settentrionale, informazione in seguito recepita nel catalogo online della biblioteca; per la datazione viene offerto un generico rimando al secolo quindicesimo. Gijsel rileva inoltre come lo stemma del dedicatario del manoscritto sia stato rimosso, rendendo la committenza non più identificabile. Infine, egli sostiene che il manoscritto sia frutto della collaborazione di due copisti, al primo dei quali attribuisce la stesura delle satire di Giovenale e di Persio e al secondo l'Ars poetica di Orazio: l'assunto è formulato però con timidezza e non senza riconoscere l'estrema somiglianza tra le due mani. ${ }^{3}$

La storia più recente del codice è ricostruita per via indiziaria, incrociando la storia delle biblioteche con le note di possesso lasciate sulle carte di guardia: la nota di acquisizione al f. 128v (TAVOLA 2) "Hic liber ad me pervenit anno 1679 ex auctione amplissimi domini van Halmale" lega il manoscritto a una famiglia di Anversa, di cui è noto in particolare il vescovo di Ypres Hendrik van Halmale, morto nel $1676 .{ }^{4} \mathrm{Nel} \mathrm{sec}$. XIX il codice passò per le mani del bibliografo belga Augustin de Backer (1809-1873: il suo ex-libris è al f. Ir), che lo cedette all'Abbazia di Drongen presso Gent, sede di un noviziato gesuita nel quale egli entrò nel 1835. Alla fine del secolo diciannovesimo, un bibliotecario del monastero, Everardus Weemaes (1809-1892), depositò la nota moralistica "Hoc manuscriptum turpissimis squalet libidinis sordibus" che si legge sulla risguardia del codice. ${ }^{5}$ Il manoscritto approdò infine nel 1959

\footnotetext{
${ }^{2}$ Sulla definizione di "paratesto": G. Genette, Seuils (Paris, 1987). Per un'applicazione del quadro teorico di Genette nella descrizione del libro manoscritto: P. Fioretti, "Sul paratesto nel libro manoscritto (con qualche riflessione sui "titoli" in età antica)", in L. Del Corso, F. De Vivo, A. Stramaglia (ed.), Nel segno del testo. Edizioni, materiali e studi per Oronzo Pecere (Firenze, 2015), 179-202.

3 Gijsel 1979 (come a n. 1), 267. La lieve differenza di tratto è impercettibile e certamente dovuta a una trascrizione in due tempi da parte dello stesso copista; ad amplificare nello studioso la percezione di un'alterità tra queste due fasi del lavoro di copia può aver contribuito la presenza di un foglio bianco (f. 115) di separazione tra la trascrizione di Persio e quella di Orazio.

${ }^{4}$ Non sono riuscito a recuperare ulteriori informazioni sulla vendita di questa biblioteca.

5 Ringrazio il sig. Luc Buffel, bibliotecario dell'Abbazia di Drongen, che mi ha comunicato via email le date di nascita e di morte di Weemaes e la data del suo ingresso nell'ordine, il 31 ottobre 1828. Secondo le informazioni a sua disposizione, Weemaes avrebbe trascorso a Drongen gli ultimi anni della sua vita.
} 
alla biblioteca dell'Istituto Sant'Ignazio di Anversa (poi UFSIA) con la cessione da parte dell'Abbazia di una sezione cospicua del suo fondo librario, diventando così patrimonio, come si è già detto, dell'odierna Biblioteca Universitaria.

Per quanto scrupolosa, l'analisi di Gijsel non ha saputo valorizzare i testi scritti sul f. 128r, che restituiscono invece informazioni decisive sulla storia più antica del codice, tanto da costituirne, come si vedrà, una vera e propria scheda anagrafica. Il foglio contiene infatti un sonetto preceduto da un'epigrafe dedicatoria. ${ }^{6}$ Eccone le trascrizioni, che eseguo sciogliendo le abbreviazioni per il latino, riportando all'uso corrente l'ortografia del volgare e introducendo l'interpunzione moderna:

Ad illustrissimum ac reverendissimum dominum dominum Fabritium episcopum camertem Federicus Veteranus urbinas excusatio.

Quel gran maestro di philosophia

Aristothile che al mondo è sì gran lume, anzi è d'ogni scientia un largo fiume, non vole ch'a molte arte uno huom se dia.

Però che ogni opra a ciò perfecta sia, richiedi tutto l'homo; et non in piume et in ocio acquista mai l'ingegno acume, et anco in troppo affanno se disvia.

Or come posso a te, digno prelato, ben satisfare in questa mia scriptura, Di giù, di su, di là, di qua intricato!

Pur, s'a te piaccia, a me fia gran ventura; et s'io vi fusse alquanto trascurato, d'ognun che scrive, questa è sua natura.

Da questi testi si ricavano l'autore e il dedicatario dei versi e, come si vedrà, lo scriba e il destinatario dell'intero codice: l'urbinate Federico Veterani e Fabrizio da Varano, vescovo di Camerino tra il 1482 e il $1508 .^{7}$

\footnotetext{
${ }^{6}$ Il sonetto non è segnalato in F. Carboni, Incipitario della lirica italiana dei secoli XV-XX (Città del Vaticano, 1982-1994).

${ }^{7}$ C. Eubel, Hierarchia catholica medii aevi ab anno 1431 usque ad annum 1503 perducta (München, 1914), 116.
} 
Copista di Federico da Montefeltro, Veterani (ca. 1450-post 1526) è personaggio ben noto a chi si occupa della cultura urbinate del secondo Quattrocento: dalla sua mano furono esemplati numerosi codici della ricca collezione del duca, ora principalmente nel fondo degli Urbinates Latini della Biblioteca Apostolica Vaticana, e suo è anche uno degli inventari più antichi della medesima biblioteca, redatto tra il 1508 e il 1517 e reso noto agli inizi del Novecento da Cesare Guasti. ${ }^{8}$ Il sonetto del codice di Anversa ne arricchisce inoltre il ben nutrito, ma poco noto, repertorio poetico, che annovera esercizi sia in latino sia in volgare, sovente lasciati di suo pugno in chiusura di codici da lui esemplati per la biblioteca urbinate: ${ }^{9}$ con un epigramma latino, egli si congeda dalla copia della traduzione dell'Iliade di Lorenzo Valla ora ms. Urb. lat. 349; con un sonetto prende commiato dal codice dei Trionfi di Petrarca con il commento di Bernardo Glicina, ora ms. Urb. lat. 351; con un epigramma latino si chiude anche il ms. Urb. lat. 984, copia dell'Historia Langobardorum di Paolo Diacono accompagnata dal De situ orbis habitabilis di Dionigi il Periegeta nella traduzione del veronese Antonio Beccaria (1400-1474) e dalla nota descrizione di Roma del così detto Anonimo Magliabechiano. Un epigramma egli aggiunse anche alla copia delle opere di Aristotele nella traduzione latina di Giovanni Argiropulo, eseguita dal fiorentino Francesco Ugolini e ora ms. Urb. lat. $1324 .{ }^{10}$ Né va tralasciato il codice Urb. lat. 736, piccola raccolta autografa di suoi

${ }^{8}$ C. Guasti, F. Odorici, "Inventario della libreria urbinate compilato nel secolo XV da Federigo Veterani bibliotecario di Federigo I da Montefeltro duca d'Urbino", Giornale storico degli archivi toscani 15.2 (1862), 127-147. Precisano la datazione dell'inventario J. Lauts, I.L. Herzner, Federico da Montefeltro, Herzog von Urbino. Kriegsherr, Friedensfürst und Förderer der Künste (München - Berlin, 2001), 244-245, 302-310. Sulla sua attività di copista: C. Stornajolo, Codices Urbinates Graeci Bibliothecae Vaticanae (Roma, 1895), XXIV-XXVIII; Id., Codices Urbinates Latini (Roma, 1902-1921), ad indicem; M. Peruzzi, Cultura potere immagine. La biblioteca di Federico di Montefeltro (Urbino, 2004), 74-76; M.G. Fachechi, "Veterani, Federico", in M. Bollati (ed.), Dizionario biografico dei miniatori italiani. Secoli $I X-X V I$ (Milano, 2004), 989; S. Fumian, "Autografia, prassi di bottega o falsificazione? Alcune osservazioni per Federico Veterani miniatore", in C. Costa, V. Valente, M. Vinco (ed.), Arte tra vero e falso (Padova, 2014), 55-66.

${ }^{9}$ Su Veterani poeta: H. Hofmann, R. Monreal, C. Schindler, "Neulateinische Dichtung am Hof von Federico da Montefeltro", Neulateinisches Jahrbuch 7 (2005), 121-181, a 126; H. Hofmann, "Literary Culture at the Court of Urbino during the Reign of Federico da Montefeltro", Humanistica Lovaniensia 57 (2008), 5-59; F. Stok, "I poeti di Federico da Montefeltro", in A. Steiner-Weber, F. Römer (ed.), Acta Conventus Neo-Latini Vindobonensis (London - Leiden, 2018), 102-125, a 105 e 117.

${ }^{10}$ A.C. de la Mare, "New Research on Humanistic Scribes in Florence", in A. Garzelli (ed.), Miniatura fiorentina del Rinascimento, 1440-1525. Un primo censimento (Firenze, 1985), App. I, 22, num. 5. 
componimenti poetici, in larga prevalenza latini, scritti per Elisabetta Gonzaga, Guidobaldo I e Federico II da Montelfeltro, Francesco Maria della Rovere e altri protagonisti di quella Urbino di inizio Cinquecento che fa da sfondo al Cortegiano di Baldassarre Castiglione. ${ }^{11}$

Infine, ai f. 365v-366r del codice Urb. lat. 405 contenente le opere di Enea Silvio Piccolomini Veterani aggiunse nel 1522 una commossa nota obituaria in ricordo di Federico da Montefeltro, seguita da due epigrammi latini e da un sonetto in volgare, i quali, sia per il ductus libero e corsivo che li caratterizza, sia per un'affinità di carattere tipologico, possono essere confrontati con i testi contenuti nel f. $128 \mathrm{r}$ del nostro manoscritto. Se la redazione del sonetto di Anversa si deve iscrivere negli anni dell'episcopato di Varano, quindi tra il 1482 e il 1508, i componimenti poetici del codice urbinate sono di almeno quattordici anni successivi: lo scarto cronologico, pur visibile in un certo irrigidimento del tratto, non impedisce tuttavia di riconoscere in modo inequivocabile la stessa mano. E qualora non bastasse la perfetta sovrapponibilità del nome di Veterani, scritto con grafia corsiva nelle epigrafi proposte a entrambi questi specimina, una più precisa analisi paleografica potrà fugare $\mathrm{i}$ dubbi residui: identici tra $\mathrm{i}$ due oggetti del confronto sono $F$ maiuscola, dove il secondo tratto tende a salire verso destra; $g$ minuscola, con l'occhiello inferiore sempre chiuso e in un solo tratto; $r$ tonda prevalentemente in legatura o a inizio parola; et con il tratto superiore che si prolunga verso l'alto; il nesso -ff- (ma lo stesso vale per -ss-/-sf-) in cui l'asta della seconda lettera tende a sovrastare la prima. Il sonetto di Anversa si può dunque ascrivere alla mano di Veterani.

Qual è il rapporto tra questo testo e il resto del codice? Come già notato da Gijsel, il bifoglio che contiene il sonetto di Veterani è ricavato da una pergamena di diversa e più scadente qualità rispetto a quella utilizzata per la realizzazione dei fascicoli contenenti i testi satirici: adatta, dunque, a fungere da guardia del manoscritto. Una guardia però che, a differenza di quella cartacea che apre il volume, doveva essere stata aggiunta agli altri fascicoli già nel Rinascimento e, come credo, contestualmente alla prima rilegatura del volume. Un'indicazione in questo senso la fornisce già Gijsel, che riconosce l'estrema somiglianza della mano che ha redatto il sonetto con quella di uno dei correttori che

\footnotetext{
${ }^{11}$ Hofmann 2008 (come a n. 9), 127, n. 26.
} 
interviene sul codice. ${ }^{12}$ Questo revisore deposita, con un inchiostro ocra molto chiaro, numerosi interventi che sono prevalentemente orientati alla verifica dei testi trascritti, per i quali egli si è servito certamente di un esemplare di collazione, ma che rivelano anche molta attenzione per la qualità estetica della trascrizione, con frequenti ritocchi e ripassi di penna, con composte correzioni su rasura e discreti interventi interlineari. È chiaro dunque che la mano di questo revisore è la stessa di chi scrive il sonetto, cioè di Veterani. Che questo bifoglio di guardia fosse già in origine solidale con il codice sembra confermato da una nota apposta in cima al f. 128r, non rilevata - o comunque non segnalata - da Gijsel. La nota è di difficile interpretazione ma la lettura condivisa da me e alcuni colleghi interpellati è "paginae in volumine 128 ", dove la cifra 2 corregge un 3 sottostante frutto forse di un errore di conto. La somma dà il numero di fogli di cui si compone il manoscritto, con la sola esclusione del f. 129 , che verosimilmente non è rientrato nel computo perché privo di testo. Chi l'ha apposta è con ogni probabilità uno dei suoi antichi possessori: a giudicare dal ductus della $g$ e della $p$ nonché dalla $e$ minuscola in due tratti con l'occhiello staccato e aperto verso l'alto, costui sembra essere il medesimo autore dei versi contenuti nello stesso foglio, che iniziano con l'endecasillabo Bello il tuo stil a guisa di Parnasso: la grafia rimanda al secolo sedicesimo e nella descrizione del codice acclusa in appendice identifica il lettore A.

La sostanziale coerenza codicologica dei fascicoli; il residuo di una mitra vescovile sullo stemma incipitario, obliterato ma ancora affiancato dalle iniziali dorate "FA"; la presenza di un componimento autografo in chiusura di volume, secondo un usus non esclusivo ma certo peculiare di Veterani; la presenza della sua mano tra gli interventi di correzione del codice: ce n'è abbastanza, a mio avviso, per formulare l'ipotesi che il codice di Anversa sia stato scritto dal copista urbinate per Fabrizio da Varano vescovo di Camerino. La valutazione della scrittura libraria di Veterani, da me condotta confrontando il codice di Anversa con il ms. Urb. lat. 1193, silloge da lui allestita per Federico da Montefeltro e contenente, tra gli altri testi, anche esametri latini, ${ }^{13} \mathrm{mi}$ sembra confer-

\footnotetext{
${ }^{12}$ Gijsel 1979 (come a n. 1), 268.

13 Il codice contiene testi in mortem di Battista Sforza e carmina per Federico da Montefeltro di Giannantonio Campano, Pandolfo Collenuccio, Francesco Filelfo, Donato
} 
mare questa ipotesi. La grafia dei due codici è infatti compatibile. In generale, si registrano lo stesso tratto tondo e elegante e scendendo nel dettaglio si apprezzano il medesimo ductus per segni complessi come $g$ minuscola, che riproduce lo stesso tratto della scrittura corsiva; \& scritto con occhiello inferiore molto marcato; il nesso -ct-, in cui la legatura forma quasi una seconda $c$ sovrastante la prima; $q$; come abbreviazione per que enclitico; infine si ritrova la $F$ maiuscola con il primo tratto orizzontale che sale verso destra, già apprezzata nella sua scrittura corsiva. Simile è anche la mise en page dei testi poetici latini: nonostante la differenza di formato dei manoscritti, la pagina è riempita grosso modo dallo stesso numero di esametri (19 nell'urbinate, 20 nell'antverpiense) e lo stesso gusto governa le realizzazioni epigrafiche apposte a mo' di intestazione dei singoli componimenti o gruppi di versi.

Il sonetto si può d'altronde inquadrare in codificate strategie di ricerca e definizione del rapporto clientelare nel Rinascimento, come quella recentemente ben studiata e descritta da Susanna de Beer per Giannantonio Campano e Pio II..$^{14}$ In questi versi Veterani dà forma a un'excusatio per una scriptura non all'altezza del destinatario, giustificando però la sua mediocre prestazione con la vita indaffarata di chi non può seguire l'ammonimento di Aristotele di dedicarsi a una sola arte. Ma che valore dare a scriptura? A una prima lettura si è tentati di interpretare il termine nel senso di "testo scritto", e quindi di riferirlo al sonetto stesso, che però così diverrebbe ad un tempo oggetto e strumento dell'excusatio. Avrebbe invece più senso riferire il sostantivo al codice, cioè all'opera di Veterani come copista, anziché come poeta. Infatti, se nel latino del Quattrocento scriptura assumeva prevalentemente il significato tecnico e filologico di lectio, nel volgare coevo non ne mancano attestazioni con il significato di "riproduzione di un testo scritto, copia". ${ }^{15}$ Alla luce degli indizi fin qui raccolti, si può dunque ipotizzare che il codice di Anversa sia stato prodotto da Veterani con l'intento di

Acciaiuoli, Martino Filetico, Giovanni Battista Cantalicio e molti altri umanisti della seconda metà del Quattrocento: Lauts-Herzner 2001 (come a n. 8), 244-245; Stok 2018 (come a n. 9), 117.

${ }^{14}$ S. de Beer, The Poetics of Patronage. Poetry as Self-Advancement in Giannantonio Campano (Turnhout, 2013), e più di recente Ead., Patronage, in Ph. Ford, J. Bloemendal, Ch. Fantazzi (ed.), Brill's Encyclopaedia of the Neo-Latin World. Micropaedia (Leiden Boston, MA, 2014), 1124-1126.

${ }^{15}$ S. Rizzo, Il lessico filologico degli umanisti (Roma, 1984), 101-104, 212-213; il Grande Dizionario della Lingua Italiana, vol. 18 (Torino, 1996), 301, riporta alla voce "scrittura" un esempio tratto da Filarete nel senso di "riproduzione di un testo scritto, copia". 
dare un saggio delle sue competenze di copista, di decoratore di codici (così va forse letta la convivenza di stili diversi nella pagina incipitaria del manoscritto), di correttore di testi e persino di poeta al nuovo vescovo di Camerino che, insediatosi nello stesso anno in cui moriva Federico da Montefeltro, e quindi in una fase di possibile incertezza professionale per il copista urbinate, si proponeva come un nuovo mecenate sulla scena marchigiana. Che il codice non sia delle migliori realizzazioni di Veterani non indebolisce l'ipotesi, anzi la rafforza: come mostrato da De Beer per il caso di Campano, l'invio di un prodotto migliore è vincolato al raggiungimento di un rapporto stabile di collaborazione e fa dunque parte della trattativa. ${ }^{16} \mathrm{E}$ Varano costituiva un interlocutore interessantissimo sia per la sua crescente influenza nel panorama del mecenatismo marchigiano di quegli anni, sia per il suo raffinato profilo culturale. È noto infatti il grande impulso che egli, sulle orme di suo zio Giulio Cesare, impresse alle arti di questa piccola signoria, complice anche la sua ascesa al soglio episcopale, che rafforzava l'esercizio del potere signorile detenuto dalla sua famiglia già dalla fine del Trecento. ${ }^{17} \mathrm{E}$ anche come umanista, Varano vantava un profilo di tutto rispetto: in contatto con importanti intellettuali della scena umbra e marchigiana del secondo Quattrocento, il vescovo di Camerino fu legato anche all'Accademia di Pomponio Leto, dove godeva di ampia considerazione se nel 1520 Giovanni Battista Pio ne raccolse una breve descrizione di Roma insieme a lavori antiquari di Biondo Flavio, di Raffaele Volterraneo e dello stesso Pomponio, non mancando di definirlo "utraque lingua cultissimus". ${ }^{18}$ Pier Francesco Giustolo, umanista spoletino allievo di Pomponio Leto, menziona Varano nelle sue egloghe (1498)

${ }^{16}$ De Beer 2013 (come a n. 14), 33.

17 G. Capriotti, "Simulacri dell'invisibile: 'cultura lignea' ed esigenze devozionali nella Camerino del Rinascimento", in R. Casciaro (ed.), Rinascimento scolpito. Maestri del legno tra Marche e Umbria (Cinisello Balsamo, 2006), 73-83.

${ }^{18} \mathrm{G}$. Conestabile (ed.), Memorie di Alfano Alfani illustre perugino vissuto tra il XV e il XVI secolo con illustrazioni e documenti inediti spettanti alla storia di Perugia e d'Italia (Perugia, 1848), 23; Capriotti 2006 (come a n. 17), 75-76. La breve descrizione di Roma, intitolata De urbe Roma collectanea, è un'epitome della Roma instaurata di Biondo Flavio: P. Victor, Pomponius Laetus, Fabricius Camers, R. Volaterranus de Urbe Roma scribentes. Flavius de locis ac civitatibus Italiae deque eius appellationibus priscis ac novis (Bononiae, ex aedibus Hieronymi de Benedictis, 1520 [USTC 800394]). Questa silloge è stata allestita a Bologna da Giovanni Battista Pio ma pubblicata solo nel 1520 da suo figlio Giulio Cesare. Il giudizio tra caporali è formulato da G.B. Pio nella lettera prefatoria ai collectanea di Varano indirizzata a Gian Matteo Giberti, suo antico allievo e potente protetto di Leone X. 
insieme ad altri pomponiani, tra cui Angelo Colocci, e gli invia una lettera (1499) di carattere filologico su alcuni luoghi del De raptu Proserpinae di Claudiano. ${ }^{19}$ Alcuni carmi latini di Varano si leggono inoltre nel codice Perugia, Biblioteca comunale Augusta, ms. C61, scritto da Francesco Maturanzio. ${ }^{20}$ Documentato è infine il suo rapporto con Aldo Manuzio, sia nell'ambito del commercio librario sia in quello filologico e editoriale. ${ }^{21}$ Se della negoziazione di un possibile rapporto clientelare si trattò, essa rimase comunque senza esito, giacché Veterani restò fino alla morte a servizio dei Montefeltro, divenendone il bibliotecario.

\section{Appendice: descrizione del codice}

Membr., s. XV ex., Italia centrale (Urbino?), mm. $179 \times 120$ (ma i fogli sono stati rifilati).

fasc. I (cartaceo, moderno $)+\left(\right.$ inc. Giovenale) $2^{10}+1^{18}+2^{10}+1^{10-1}+3^{10}+$ (inc. Persio) $1^{10}+1^{8}+$ (inc. Orazio) $1^{10}+$ (sonetto di Veterani) II.

Numerazione moderna a matita sul margine superiore destro dei fogli. La rilegatura è costituita da piatti di cartone rivestiti di pelle di porco; sulla costa si legge una vecchia segnatura "Manu-|scriptus $\mid 310$ ", che è stata lavata e sostituita, con inchiostro più scuro, da "Iuvenalis | et | Persius. | MS | Horat. | Ars | poet." Taglio colorato in rosso.

f. 1r-97v, Iuvenalis, Saturae; f. 98r-115v (f. 115r-v bianco), Persius, Saturae; f. 116r-127v, Horatius, Ars poetica, seguita, in cinabro e quasi

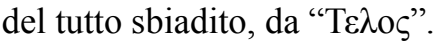

La pagina incipitaria presenta tre diverse tipologie di decorazione: nel bordo superiore si trovano elementi fitomorfi con al centro un anello dorato; il bordo destro e quello inferiore sono decorati a bianchi girari, mentre il bordo sinistro presenta un'asta dorata intorno alla quale si avvita una greca blu. Lo stemma del committente, contenuto in un clipeo su campo rosso delimitato da una ghirlanda, è stato rimosso quasi del tutto. Oltre a qualche traccia di colore oro e blu, si distinguono ancora una mitra vescovile bianca e le due lettere dorate $F$ e $A$ ("Fabricius"?)

${ }^{19}$ F. Calitti, "Giustolo, Pier Francesco", in Dizionario biografico degli Italiani, vol. 57 (Roma, 2001), 384-387.

${ }^{20}$ G. Zappacosta, Studi e ricerche sull'umanesimo italiano (Bergamo, 1972).

${ }^{21}$ Capriotti 2006 (come a n. 17), 76, che si rifà al volume, per me irraggiungibile, di F. Pecchi (ed.), B. Feliciangeli, Autobiografia di un orafo bolognese del Cinquecento (Camerino, 1913). 
rispettivamente sul lato destro e sinistro dello stemma. Le lettere iniziali sono dorate e si stagliano su un campo quadrato, colorato in verde e/o in blu e decorato con filigrane in oro; la prima lettera incipitaria presenta anche una bordura laterale in rosso. Le iscrizioni contenenti l'autore e il titolo delle opere e quelle che ne introducono le singole satire sono sempre in blu.

Sulla controguardia cartacea del codice è incollata un'etichetta con la segnatura di collocazione del Noviziato dei Gesuiti di Drongen, in Belgio: "Bibl. D. P. Trunc.| H.2.T.99", cassata da ripetuti segni di penna. Sullo stesso foglio, a piena pagina "Hoc manuscriptum turpis-|simis squalet libidinis | sordibus | E. Weemaes". Al f. Ir, dall'alto al basso: "A. de Backer"; timbro viola dello stesso noviziato di Drongen "Bibl. Magistrorum Dom. Trunc. S.J.", con al centro una successiva collocazione del codice scritta in matita "b.4". Ancora in matita, segnatura di collocazione dell'UFSIA "P 1/1". A mezza pagina, un cartiglio bianco riporta in penna blu e in nederlandese una recente nota di biblioteca: "Over dit handschrift werd een grondige studie gepubliceerd door prof. J. Gijsel (Ufsia) in: Scriptorium T 33 (1979), p. 266-270 | opgenomen op $16 \mathrm{~mm}$. film." 22 In fondo alla pagina è presente un timbro della Biblioteca dell'Istituto Sant'Ignazio di Anversa con un numero di inventariazione a penna " 19583 ".

Il bifoglio $128 \mathrm{r}-129 \mathrm{v}$ è una guardia membranacea. Al f. $128 \mathrm{r}$ (TAVOLA 2), un sonetto autografo di Federico Veterani, inc.: "Quel gran maestro di philosophia". Seguono sette endecasillabi in italiano di altra mano (= A), aggiunti successivamente, l'ultimo dei quali è riscritto su rasura e difficilmente leggibile, inc.: "Bello il tuo stil a guisa di Parnasso"; f. 128v, "hic liber ad me pervenit anno 1679 ex auctione amplissimi domini van Halmale" e più oltre, per mano del lettore A, "quid ne diceres plures pontes Romae quam homines boni"; appunti di A anche a f. 128r: "crētâtā āmbitio" (Pers. 5.176, prova di scansione prosodica); "farrago libelli" (Iuv. 1.81); "omne in praecipitium vitium stetit" (Iuv. 1.149); "rara est concordia famae atque pudicitiae" (Iuv. 10.293); "Senuerunt Iuppiter et Mars / porticibus" (Iuv. 6.59); "nemo in se se tentat" (Pers. 4.23).

KU Leuven

fabio.dellaschiava@kuleuven.be

22 "Su questo manoscritto è stato pubblicato uno studio dettagliato dal prof. J. Gijsel (Ufsia) in: Scriptorium T 33 (1979), p. 266-270 | impresso su un microfilm da 16 mm." 


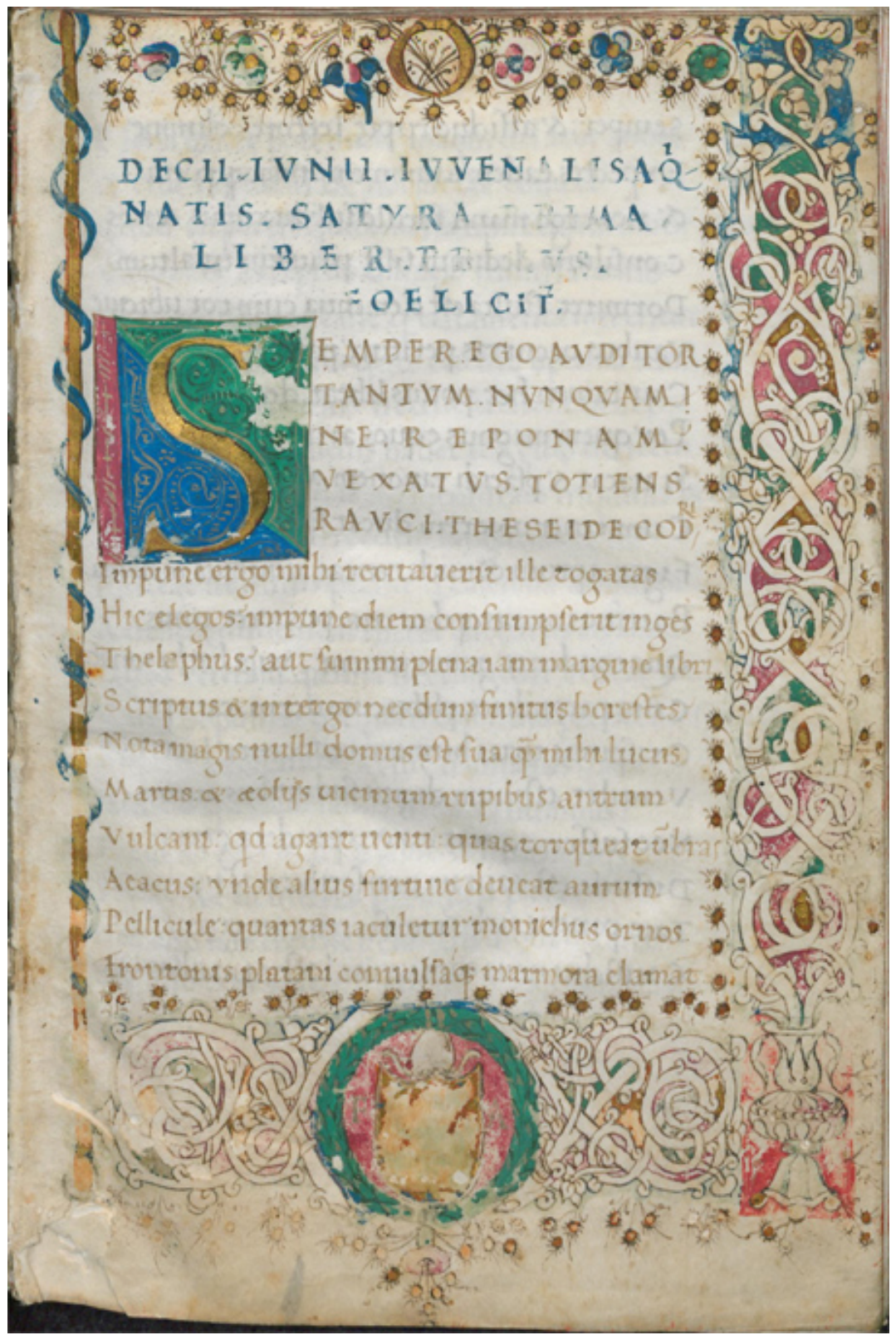

TAvola 1: Antwerpen, Universiteitsbibliotheek, Bijzondere Collecties, MAG-P 60.5, f. 1r. Frontespizio con lo stemma parzialmente rimosso di Fabrizio da Varano. 


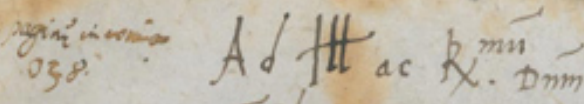

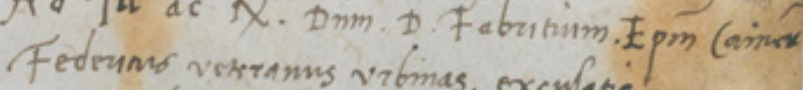
Qnel gran marftoo an philofoplin a ruforble cod mondo es sman lume Amzi $e, \operatorname{dogm}$ scuentia vn lazgo finme Non vole chamole aree uno bion

$P$ ero th oom opa a cio 250 huom fe did Rimied mito thomo:

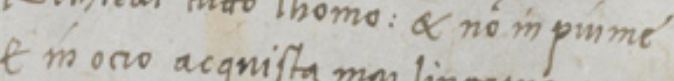
t morro acgnifta mm lingegno arnme C onco in roppo affimo se difiria. o r comé poffo a te diono pulato Bensatifane in $\tilde{g}$ for mia sriphras Deom disu dila di giw mirieato Pur $3 \pi$ te piaccia, a méfia man notum. e sue mifusse alopto trajmzato Doommon or saine fra

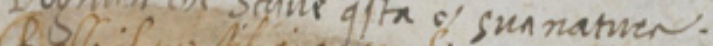
pels il tiw lat a givisa di parrasso

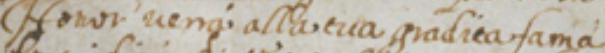
te sidisa di t'non qua matrele

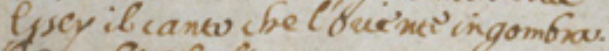

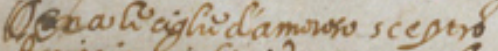

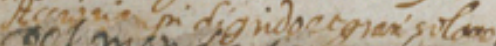
scamondo suxd vinivofe ac.

TAVola 2: Antwerpen, Universiteitsbibliotheek, Bijzondere Collecties, MAG-P 60.5, f. 128r. Sonetto autografo di Federico Veterani. 